\title{
Grief in children and adolescents: a review
}

\author{
Pia Sanghvi \\ Independent Mental Health Professional / Psychotherapist, Mumbai \\ Corresponding Author: Pia Sanghvi \\ E-mail: piasanghvi.therapy@gmail.com (www.linkedin.com/in/piasanghvi).
}

\begin{abstract}
This review article covers the various facets of grief in children and adolescents. The aim is to provide a framework for professionals to enable holistic healing after experiencing a loss. Within this review, different types of responses and theoretical models on the subject have been examined. Due to widespread trauma concerns in our country and within specific populations, traumatic grief in children and common trauma-related symptoms has been included. Lastly, evaluation considerations as well as evidence-based interventions and approaches for grief concerns are discussed.
\end{abstract}

Keywords: grief, children, adolescents, holistic, healing, trauma, traumatic grief.

(Paper received $-25^{\text {th }}$ June 2019 , Peer review completed $-9^{\text {th }}$ August 2019)

(Accepted $-12^{\text {th }}$ August 2019)

\section{INTRODUCTION}

In spite of grief being a universal, human experience, it is a topic that is often overlooked. Children and adolescents are exposed to death, loss and grief in various ways. From losing a favorite toy to the death of a family member, they can experience a wide range of losses through their childhood. Loss is a fact of existence that usually finds it's way into most our lives. Therefore, instead of thinking about how we can protect the young from these experiences, let us think about how we can understand, empathize, and support them as they cope with their emotions. In this article, you will gain an understanding of grief and mourning through the work of various pioneers and theoretical models on the subject. Additionally, different possible responses to loss as well as relevant evaluation considerations and treatment approaches are discussed.

\section{What is Grief}

An initial definition provided by William Worden stated grief as 'an experience of loss due to the death of a loved one' [1]. He then went on to broaden the existing definition to include other losses as well. Grief has been described as a natural, multi-faceted, personal response to loss. Initially, it was thought to solely impact our emotional wellbeing however today we know that such an experience can also affect our physiological, cognitive and behavioral functioning.

Bereavement has been described as a state that includes a range of grief and mourning processes [2], while mourning encapsulates the process by which grief is resolved [3]. It has been found that the intensity and duration of a response would depend on a myriad of factors such as nature of attachment, circumstances surrounding the loss, personal history, personality variables, and coping style. Therefore, the process often differs from individual to individual. 


\section{Types of Grief}

Uncomplicated Grief: Uncomplicated bereavement comprises of a wide range of physical sensations, thoughts, emotions and behaviors that commonly present after a loss. Common expressions include shock, thinking about the person, sadness, anger, fatigue, loneliness, changes in sleep and appetite, and many more that have been listed below. The child or adolescent is able to heal, integrate the loss and complete the mourning process.

Complicated Grief: Complicated grief involves not being able to adapt to the loss, having difficulty managing daily tasks and a delay in returning to prior functioning. Children or adolescents could have responses similar to those who experience uncomplicated grief. However, the intensity and duration of the experience could be greater. In complicated grief, there is something that impedes the process of integrating the loss and moving forward with life [4]. It has also been found that in some cases, complicated grief is often associated with trauma and depression.

The four types of complicated grief reactions are: [5]

a) Chronic Grief Reactions - These grief reactions are prolonged and continue for considerable time after the loss. For example: An adolescent continues to experience and shows signs of grief four years after losing a close friend. In such cases, it is important to understand what remains unresolved in the grieving process.

b) Delayed Grief Reactions - Suppressed, postponed or inhibited grief responses are common when the individual has not been able to process the loss when it occurred. Instead, expressions of grief appear at a much later period. Hence, it is often misattributed and can be neglected or dismissed. For example: A child may not comprehend the death of his sibling. S/he may process the loss a few months later experiencing an overwhelming delayed grief reaction.

c) Exaggerated Grief Reactions - These grief reactions are observed when the individual is overwhelmed by grief and experiences additional symptoms of depression, anxiety, phobias, posttraumatic stress disorder or substance abuse. For example: A child witnesses the death of his/her parent and experiences trauma-related symptoms.

d) Masked Grief Reactions - In this type of grief response, physical symptoms may begin manifesting. It must be noted that physical conditions present prior to the loss could also increase in intensity due to additional stress on the brain and body. Psychosomatic complaints are commonly seen in children and adolescents as a method to cope. For example: An adolescent may develop recurrent headaches after losing a family member.

\section{Childhood Traumatic Grief}

Cohen and Mannarino [6] define Childhood Traumatic Grief (CTG) as a condition in which trauma symptoms overwhelm and interfere the natural grieving process [7]. CTG generally arises when a child experiences a loss that is traumatic in nature (e.g. loss during a natural disaster, death by accident). However, it can also occur in the case of gradual, anticipatory losses (e.g. terminal illness, death due to old age). The experience of trauma can lead to complicated mourning [5] and the development of continued post-traumatic stress. Cohen and Mannarino [6] listed common symptoms of trauma seen in children:

1) Re-experiencing: The child or adolescent has distressing memories, recurrent thoughts and dreams of the deceased or traumatic event. Young children may reenact parts of the event through play.

2) Avoidance: The child may begin to withdraw and move away from places, memorabilia or situations that remind him/her of the event and/or person.

3) Hyperarousal: Physical symptoms such as increased anger, anxiety, difficulty sleeping and restlessness are often observed.

4) Emotional, behavioral dysregulation or distressing thoughts: The child or adolescent may have trouble regulating his/her emotions and behaviors. They may cry frequently, appear zoned out, or have outbursts of anger. Distressing thoughts focusing on self-blame and omen formation are often noticed.

5) Learning problems: They may have trouble with cognitive processes such as attention, memory, speed of processing etc. that interfere with their daily activities. 


\section{The Grieving Process}

\section{Kübler-Ross's Five-Stage Model}

Elisabeth Kübler-Ross [8] introduced a five-stage model of grief in her book 'On Death and Dying'. The model was created based on the experiences of terminally ill patients and people who lost a loved one. It was later expanded to include major and minor losses such as loss of a relationship or loss of insurance. The stages of this model do not fall into sequential order. Kübler-Ross believed that a person experiences at least two of the five stages and move from one stage to another, several times. The five stages of grief are as follows -

1) Denial: This stage involves conscious or unconscious refusal to accept the reality of the situation.

2) Anger: The child or adolescent may experience and perhaps express the emotion. It could be directed inwards or towards the deceased, a family member, teacher or healthcare worker.

3) Bargaining: Known as the negotiation stage, bargaining provides temporary escape from the situation and instills hope. People often pray, practice rituals, make deals or promises in an attempt to reverse the circumstances of the situation.

4) Depression: During this stage, a sense of finality is processed. People experience symptoms of depression such as hopelessness, loss/increase of appetite, sadness, frequent crying etc.

5) Acceptance: The acceptance stage is the final stage wherein individuals begin to accept the reality of the situation i.e. the fact that death is inevitable or that it has taken place.

Let us discuss these stages of grief using an example. A child may try to convince himself that his parents are having a fight and not actually going to divorce each other (Denial). The child may then experience anger towards one or both his parents for breaking up the family (Anger). In the next stage, the child begins to 'bargain' in an attempt to avoid coming to terms with the situation. S/he may think, "If I behave like a good child, my parents will not fight and they will be together" (Bargaining). Once the child realizes that the divorce is going through, s/he may experience sadness or dysphoria (Depression). In the last stage, the child accepts the reality of the divorce and tries to adjust to the new situation (Acceptance).

The Kübler-Ross model was initially developed to help terminally-ill patients and bereaved adults. However, the concept provides a valuable framework to understand the process of grief in children and adolescents.

\section{Tasks of Mourning}

William Worden [5] used the task model to describe the mourning process. He believed that the concept emphasized on action and intervention. The model emphasizes on attending to four tasks in order to pass through the mourning process. Tasks can be revisited and worked through from time to time. An individual can be working through more than one task at a time. The mourning process is believed to be complete when the individuals integrate the loss into their lives, and ground themselves in the present.

Task I - To Accept the Reality of the Loss: The first task of grieving is to become aware and conscious of the death or loss. There are numerous ways in which people protect themselves. One such way is denial. Worden stated that some other ways reality can be avoided is when mourners alternate between 'believing' and 'disbelieving'. In such situations, it can be suggested that the person help with funeral procedures and rituals to help process the event, become oriented to reality and begin their healing journey.

Task II - To Process the Pain of Grief: The type of pain depends on many factors unique to the loss. Feelings of anger, guilt, loneliness, anger or depression are commonly addressed during this period. The incompletion of this task results in 'not feeling' or numbness. People begin to avoid painful thoughts, cut off from their feelings and deny the pain altogether. It is necessary to work through the pain as it can manifest in other forms such as physical symptoms or continued dysregulation of the nervous system.

Task III - To Adjust to a World Without the Deceased: The third task involves making external, internal and spiritual adjustments after the loss. External adjustments relate to how the loss affects everyday functioning such as a change of environment. Internal adjustments are associated with how the death affects sense of self such as one's self-esteem and core beliefs. Spiritual adjustments are those, which affect people's assumptions and opinions about the world, cultural beliefs and values. 
Task IV - To Find an Enduring Connection with the Deceased in the Midst of Embarking on a New Life: In this task, the survivor must develop ways to continue the bond with the deceased. For example, finding a way to memorialize the loss. Acceptance at this stage does not mean that the relationship with the deceased has ceased to exist. It is being able to find a unique and personal way of continuing the connection in a healthy manner. Goals of this stage include discovering new aspects of oneself, forming relationships with others, regaining an interest in life, finding hope and most importantly, functioning.

\section{The Good Grief Program Model}

Sandra Fox developed the Good Grief Program Model during the 1980's [9]. The program was first discussed in her book, 'Good Grief: Helping Groups of Children Deal with Loss When a Friend Dies'. The model is similar to that of Dr. William Worden's in the sense that it talks about tasks of mourning. The Good Grief model was created to understand grief in children and adolescents. The four tasks of mourning in this model are:

- Understanding: This task involves making sense of the concept of death, and the death of the particular person. The child or adolescent begins to grasp that the person is no longer alive and will not continue being part of his/her life. Depending on the age and developmental stage of the young person, an explanation should be given. There are three factors that could interfere with the completion of this task. The first one is the way death is defined, the second is constant development of the child's understanding of the concept and the third is magical thinking. Clinicians could address each factor to aid the healing process.

- Grieving: This phase involves working through various feelings that are part of the mourning process. The child or adolescent must be made to understand that feelings of sadness and anger are normal grief responses. Clinicians should note that there is no right way of grieving. The style of grieving depends on factors such as age, developmental stage, personality characteristics, relationship with the deceased and circumstance surrounding the death. Grieving is an individualistic process and will differ from person to person. For example, adolescents often find it comfortable to grieve with their friends rather than their families. In that case, family members should let them know they are there for them if they need support or a safe space to communicate.

- Commemorating: Within this stage, one begins to find a way to remember the deceased and memorialize the loss. For example, the child may ask to keep an object of the deceased to remember them by. Adolescents could be encouraged to participate in formal or informal rituals. These activities convey the value of life, reality of the death and help with the integration process.

- Going on: Once the tasks of understanding, grieving and commemorating have been completed, children and adolescents often look for permission from significant others that it is okay to go on with life. In that case, one could discuss that 'going on' need not mean 'forgetting'. If a child or adolescent is unable to function or continues to feel overwhelmed by the loss, the tasks could be reviewed in reverse order. The clinician must find out at what stage something remains unresolved. In Dr. Sandra Fox's words, this is how 'good grief' can be achieved.

\section{Evaluating Grief in Children and Adolescents}

Expressions of Grief: This shall vary from individual to individual. Hence, it is important for clinicians to understand that there is a range of grief responses, and to assess whether the child/adolescent is progressing adaptively through the mourning process or having difficulties. The intensity and duration is variable and determined by various factors such as coping mechanisms, attachment style, personality variables, access to a support system and so on. Listed below are common responses to grief -

- Sadness, crying

- Feelings of emptiness and loss

- Frequent somatic complaints such as headaches or stomachaches

- Muscle tension, tremors

- Appetite changes (eating more or less)

- Regressive or impulsive behaviors

- Sleep disturbances (nightmares, insomnia) 
- Attention difficulties, forgetfulness, confusion

- Fatigue or low energy

- Preoccupation with thoughts of the loss

- Restlessness or hypervigilance

- Fears, phobias, obsessions, compulsions

- Loneliness or fear of being alone

- Anxiety, worry or uneasiness

- Increased irritability

- Shock, denial or numbness

- Yearning for the deceased

- Excessively imitating or searching for the deceased

- Loss of interest in daily activities

- Decline in academic performance

- Self harm or suicidal ideation

- Dissociating

- Guilt or self-reproach

- Feelings of anger

- Withdrawing from others

Diagnosis: There is much debate about diagnosing complicated grief in children and adolescents. Several researchers have mentioned that there is a need for more research on grief responses in this population. Some suggest that the criteria should take into account childhood development while others believe that the criteria should remain similar to that of adults. The DSM-5 [10] proposed criteria for a bereavement diagnosis known as 'Persistent Complex Bereavement Disorder' (PCBD). The proposed criteria stated that children after the age of 1 year could be diagnosed with this disorder if concerns continue to persist 6 months post the death of a loved one. The DSM mentions that certain symptoms present themselves differently in children than in adults. For example: yearning for the deceased may manifest in behavior or themes of play. However, the Task Force of the DSM-5 decided that there was a dearth of evidence to warrant an inclusion of the disorder as an official diagnosis and hence placed it under Conditions for Further Study.

The presence of Post-Traumatic Stress Disorder (PTSD), symptoms of depression and anxiety in childhood traumatic grief has been researched and determined. Please note that depression, anxiety and trauma-related symptoms are commonly seen in childhood traumatic grief. Hence, it is important that clinicians assess as well as monitor the intensity and duration of these symptoms to determine if they meet the criteria for a condition. In addition to the above, the DSM-5 contains a footnote, which instructs clinicians to carefully determine whether the client is experiencing grief or fulfills the criteria for having a 'Major Depressive Episode' (MDE). It also covers 'Uncomplicated Bereavement' under conditions that may require clinical attention or affect a person's mental health. It is common for individuals to experience grief after a loss impacting one's over health and functioning. Hence, it is necessary to obtain a detailed case history and take bereavement under consideration while conducting an evaluation.

\section{Treatment}

Loss brings about a broad range of grief responses as noted above. Most children and adolescents are able to cope with the loss and complete the mourning process. However, there are some who require the help of clinicians to provide support as they adjust to the loss.

Early interventions are advisable in situations when:

- the loss is traumatic or sudden in nature

- the loss is significant to the child or adolescent (e.g. sibling, parent etc.)

- the nature of relationship with the deceased was conflicted

- the signs and symptoms that appear are severe

- mental health concerns were present prior to the loss 


\section{Grief Therapy}

Therapy is an effective way to learn how to cope with the stress associated with loss. Worden [5] differentiated between grief counseling and grief therapy. Grief counseling involves helping individuals work through uncomplicated grief reactions. Grief therapy, on the other hand, is conducted when the individual is not able to come to terms with the loss i.e. they have complicated grief reactions. Each experience of grief is unique, personal and complex, hence therapists must tailor interventions to meet the specific needs of the client. The goal of grief therapy is to help children and adolescents understand and cope with their grief, helping them adjust to a new reality and integrate the loss into their lives. In therapy, one must identify and resolve the conflicts that interfere with the process of mourning. Worden [5] provides certain useful techniques that therapists can use for grief therapy. These techniques are -

a) Writing: Letter or journal writing can be used to express thoughts and emotions regarding the loss. Writing can help address things that were left unsaid. This gives the individual, a chance to freely express which helps in healing.

b) Drawing: Drawing too, provides a non-verbal means to express oneself in therapy. Drawings are less susceptible to defenses than talking. Hence, the therapist can gain insight into the child or adolescent's unconscious or repressed thoughts and feelings through this method.

c) Role-Playing: An effective tool to help clients adjust to internal and external adjustments after a loss. The therapist can support the child to express, play out certain situations and build skills such as boundary setting through this method. This can aid in adjusting to a new reality.

d) Cognitive Restructuring: In this technique, the therapist challenges unhelpful thoughts associated with the loss or event. The goal with cognitive restructuring is to help the child to identify these thoughts and replace them with healthy patterns of thinking.

e) Memory Books: A memory book can pose as a visual and concrete aid containing photographs, poems, stories about the loved one, and other such memorabilia. This activity helps the child/adolescent to reminisce and mourn the loss. The memory book can be added to and used to reintegrate the loss as they grow and develop.

f) Directed Imagery: Visualization often helps with individuals who have experienced a loss. Techniques such as empty chair or conversations with the deceased can be used to resolve unfinished business. Brown [11] provides guided imagery techniques for grief concerns.

g) Metaphors: Metaphors are often useful to talk about the subject of loss without using a more directive approach on the subject. A useful tool to lowering the resistance of pain in situations when clients are unable to verbalize or make sense of their feelings or thoughts.

\section{Grief Support Groups}

Support groups can be used with children and adolescents to address grief-related concerns. Groups should be constructed taking into factors such as the child/adolescents needs, developmental stage and the nature of loss. Basic guidelines clinicians can follow to form and conduct grief support groups [12]-

- Draft a timeline and create a plan

- Obtain parent/guardian permission

- Provide a safe space to explore and express

- Complete the goal setting process

- Conduct developmentally appropriate activities in line with tasks of grief

- Address thoughts, emotions and ideas

- Provide multiple avenues for expression

- Discuss, practice and model age-appropriate coping skills

- Be sensitive and respect religious and cultural influences

- Be flexible, accept variations in children's grief

\section{Art Therapy}

Art therapy is the therapeutic use of art within a professional relationship, by people who experience mental health concerns, trauma or major life changes [13-14]. It is an expressive therapy form that uses the 
creative process of making art to improve a person's physical and psychological wellbeing. Any type of art medium such as drawing, painting, sculpting, digital art or photography can be employed within the therapeutic process. Art therapists are trained to pick up on certain non-verbal cues that are expressed during the creative process. This therapy approach can be used with a diverse range of concerns. It has been useful in healing trauma and grief in children/adolescents.

\section{Play Therapy}

Play therapy is a well-established and popular approach for children and adolescents. The use of play has many therapeutic benefits that encourage children to communicate either verbally or non-verbally. In cases where the child or adolescent is displaying resistance or has difficulty expressing their emotions, play has been found to be effective. This therapy approach can be divided into two basic types - directive and nondirective. Directive play therapy involves using structure and guidance by the therapist to help the child work through emotional and behavioral concerns. Whereas, non-directive or unstructured play consists of using free play within certain therapeutic conditions. This therapy approach is commonly used to address grief in children and adolescents [15].

\section{Bibliotherapy}

Bibliotherapy is a therapeutic approach that uses literature to improve mental health. The concept is based on the fact that humans tend to identify with characters in literary expression. This therapy method consists of selecting reading material relevant to the client's concern. Sharing carefully selected stories and picture books with children can help them cope with challenging experiences such as loss of a loved one. Bibliotherapy can be of two types - fictional and non-fictional. Books can also be used to psycho-educate parents about concerns relating to their children or adolescents. This approach is also a common intervention technique in Cognitive-Behavior Therapy [16].

\section{Trauma-Focused Cognitive Behavioral Therapy}

Trauma-Focused Cognitive Behavioral Therapy (TF-CBT) is an evidence-based treatment method developed by Judith Cohen and other professionals. It is a short-term treatment approach that has been found to be effective in addressing trauma-related concerns. This model was primarily developed for children and adolescents who have trauma-induced symptoms such as depression, anxiety, post-traumatic stress or display behavioral problems post a loss. TF-CBT has been used with children who were bereaved by causes such as community or domestic violence, suicide, military deaths, terrorism, disasters, accidents and illnesses. Numerous studies have proven the efficacy of this treatment model with diverse, crosscultural populations, specifically with children and adolescents. A study conducted by researchers in Japan found that TF-CBT was feasible for treating trauma-related concerns in Asian children [17-18].

The components of the TF-CBT treatment model are summarized by the acronym 'P.R.A.C.T.I.C.E'. As each component is completed, the child or adolescent will move on to the next component in the treatment plan. A core part of the therapy approach is the use of gradual exposure for trauma, at each component stage. Through this children/adolescents gradually work through healing the trauma. The components of the treatment method are described below:

- Psychoeducation: Psychoeducation involves providing information to the family regarding the child's mental health and treatment plan. This is continued through the course of treatment.

- Parenting component: In TF-CBT, sessions are conducted with the parents as well as the children. Along with the nine components, interventions related to parenting skills are conducted.

- Relaxation skills: Relaxation skills taught to both parent and child help to regulate and manage trauma-related symptoms. Skills such as yoga, mindfulness, deep breathing, progressive muscle relaxation and so on, are the focus.

- Affective modulation skills: Affective modulation skills are tailored so that they are individualized for the parent and child. Children and parents are taught how to regulate feeling states through identification, normalizing conflicting emotions, and learning strategies.

- Cognitive coping skills: Therapists encourage the child and parents to explore their emotions, thoughts and behaviors relating to upsetting situations. They learn how to gain control over their 
thoughts followed by feelings and behaviors. They also learn how to identify unhelpful thoughts replacing them with adaptive or helpful thoughts.

- The trauma narrative and cognitive processing of trauma experiences: After skill- building, the children are asked to create a trauma narrative with the therapist. This narrative could be in the form of a song, book, poem or any other method of expression. The purpose of this component is to identify trauma-related cognitions and create a safe space for emotions, thoughts.

- In vivo mastery of trauma reminders: Specific trauma cues are worked upon through graduated exposure. For example: A child who was bullied in the playground of the school may not wish to play there anymore. This can be resolved through the process of gradual exposure to reminders of the traumatic experience.

- Conjoint child-parent sessions: Joint sessions are taken with the parent and child to facilitate communication about the experience, increasing validation and support received.

- Enhancing safety and future developmental trajectory: Safety skills for specific concerns are taught to the parent and child. They can be practiced within the joint or individual sessions. For example: Bullying safety skills can be taught to the child.

\section{Conclusions}

As a society, and as clinicians it is important that we gain an in-depth understanding of the impact and role of loss and grief. Mental health professionals have an opportunity to help children and adolescents make sense of, cope and heal after such an experience. It is important to note that each individual's process is unique and intervention may be required when quality of life and functioning is affected.

\section{REFERENCES}

1. Bonanno GA, Kaltman S. The varieties of grief experience. Clin Psychol Rev 2001;21(5):705-34.

2. Nader K, Salloum A. Complicated grief reactions in children and adolescents. J Child Adolesc Trauma 2011;4(3):233-57.

3. Jacobs S. Traumatic grief: Diagnosis, treatment, and prevention. Psychology Press; 1999.

4. Dyregrov A, Dyregrov K. Complicated grief in children-the perspectives of experienced professionals. OMEGA J Death Dying 2013;67(3):291-303.

5. Worden JW. Grief counseling and grief therapy: A handbook for the mental health practitioner. Springer Publishing Company; 2018.

6. Cohen JA, Mannarino AP. Supporting children with traumatic grief: What educators need to know. School Psychol Int 2011;32(2):117-31.

7. Crenshaw DA. An interpersonal neurobiological-informed treatment model for childhood traumatic grief. OMEGA J Death Dying 2007;54(4):319-35.

8. Kübler-Ross E, Kessler D. On grief and grieving: Finding the meaning of grief through the five stages of loss. Simon and Schuster; 2005.

9. Fox GC, Reid GE, Salmon A, Mckillop-Duffy P, Doyle C. Criteria for traumatic grief and PTSD. Br J Psychiatry 1999;174(6):560-6.

10. Fox J, Jones KD. DSM- 5 and Bereavement: The Loss of Normal Grief?. J Couns Dev 2013;91(1):113-9.

11. Brown JC. Loss and Grief: An Overview and Guided Imagery Intervention Model. J Ment Health Couns 1990;12(4):434-5.

12. Tonkins SA, Lambert MJ. A treatment outcome study of bereavement groups for children. Child Adolesc Soc Work J 1996;13(1):3-21.

13. Case C, Dalley T. The handbook of art therapy. Routledge; 2014.

14. Beaumont SL. Art therapy for complicated grief: A focus on meaning-making approaches. Can Art Ther Assoc J 2013;26(2):1-7.

15. Stutey DM, Helm HM, LoSasso H, Kreider HD. Play therapy and photo-elicitation: A narrative examination of children's grief. Int J Play Ther 2016;25(3):154-8.

16. Briggs CA, Pehrsson DE. Use of bibliotherapy in the treatment of grief and loss: A guide to current counseling practices. Adultspan J 2008;7(1):32-42.

17. Cohen JA, Mannarino AP. Trauma-focused CBT for traumatic grief in military children. J Contempor Psychother 2011;41(4):219-27. 
18. Cohen JA, Mannarino AP, Staron VR. A pilot study of modified cognitive-behavioral therapy for childhood traumatic grief (CBT-CTG). J Am Acad Child Adolesc Psychiatry 2006;45(12):1465-73.

$* * * * * * * * * * * * * * * * * * * * * * * * * * * * * * * * * * * *$

\author{
Acknowledgements - Nil \\ Conflict of Interest - Nil \\ Funding - Nil
}

